\title{
Non-self-averaging in autocorrelations for Potts models on quenched random gravity graphs
}

\author{
Wolfhard Janke † and Desmond A Johnston $\ddagger$ \\ $\dagger$ Institut für Theoretische Physik, Universität Leipzig, Augustusplatz 10/11, D-04109 Leipzig, \\ Germany \\ ‡ Department of Mathematics, Heriot-Watt University, Edinburgh, EH14 4AS, Scotland
}

Received 30 November 1999

\begin{abstract}
We investigate the non-self-averaging properties of the dynamics of Ising, 4-state Potts and 10-state Potts models in single-cluster Monte Carlo simulations on quenched ensembles of planar, trivalent $\left(\Phi^{3}\right)$ random graphs, which we use as an example of relevant quenched connectivity disorder.

We employ a novel application of scaling techniques to the cumulative probability distribution of the autocorrelation times for both the energy and magnetization in order to discern non-selfaveraging. Although the specific results discussed here are for quenched random graphs, the method has quite general applicability.
\end{abstract}

\section{Introduction}

The effect of quenched, typically bond, disorder on the critical behaviour of spin systems has been a subject of interest for many years [1], both because of its own intrinsic interest and the prevalence of disordered systems in nature. The Harris criterion states [2] that the critical behaviour of a pure system will be unchanged by the introduction of weak quenched bond disorder if the specific-heat exponent of the pure system, $\alpha_{p}$, is less than zero. By the same token if $\alpha_{p}$ is greater than zero the disordered system will not be governed by the pure fixed point, but rather a new disordered fixed point. The borderline, $\alpha_{p}=0$, constitutes a marginal case which requires more careful investigation for each specific case.

As was emphasized in [3], a pure fixed point is usually characterized by a Gaussian distribution of renormalized couplings around the fixed point Hamiltonian which tends to a delta function in the thermodynamic limit, whereas a disordered fixed point might be expected to be characterized by some other distribution which tended to a finite width in the thermodynamic limit $\dagger$. For a fixed point with a finite width distribution of couplings a measurement of the density of an extensive thermodynamic quantity such as the energy $E$, magnetization $M$, specific heat $C$ or magnetic susceptibility $\chi$, would be different on each sample because of the different disorder realizations. Such behaviour is called non-self-averaging.

This non-self-averaging behaviour for an observable $X$ (such as $E, M, C$, or $\chi$ above) can be characterized by examining the normalized variance

$$
R(X)=\frac{\left[X^{2}\right]_{\mathrm{av}}-[X]_{\mathrm{av}}^{2}}{[X]_{\mathrm{av}}^{2}}
$$

$\dagger$ As we note in what follows, the presence of such a finite width distribution, and hence non-self-averaging, is not a hard and fast indicator of new, random fixed points since Poisonnian random lattices still display pure critical behaviour even though they also display non-self-averaging properties. 
where [... $]_{\text {av }}$ denotes an average over the disorder realizations [3-5]. If $R(X) \rightarrow 0$ as $N \rightarrow \infty$, where $N$ is the number of lattice sites, then we have self-averaging, whereas if $R(X) \rightarrow c$, with $c$ a constant, we have non-self-averaging. Self-averaging systems may in turn be divided into strongly self-averaging systems where $R(X) \sim N^{-1}$, which is the typical behaviour off criticality and at pure fixed points and weakly self-averaging systems where $R(X) \sim N^{\kappa}$ with $-1<\kappa<0$, which has been observed for the case of irrelevant quenched disorder at criticality [4]. For self-averaging systems measurements on a single large system are sufficient, whereas for non-self-averaging systems measurements on different realizations of the disorder must be carried out in order to obtain reliable ensemble averages.

In this paper we will be concerned with quenched connectivity disorder, which has received rather less attention than quenched bond disorder. The possible influence of quenched geometrical disorder (connectivity, aperiodicity, ...) on the universality properties of statistical mechanical systems in general has been explored by Luck [6] who arrived at a criterion rather similar in spirit to the Harris criterion for the (ir)relevance of such disorder. He noted that if $B(\Omega)$ was the number of bonds in a region $\Omega$ and $\Sigma(\Omega)=\sum_{\langle i j\rangle \in \Omega} J_{i, j}$ was the sum of bond values in that region then, although both these quantities scaled as the volume $N$ of the region $\Omega$, one had

$$
\Sigma(\Omega)-J_{0} B(\Omega) \sim N^{\phi}
$$

where $J_{0}$ was the limiting value as $N \rightarrow \infty$ of $\Sigma(\Omega) / B(\Omega)$ and $0 \leqslant \phi<1$ was a fluctuation (or wandering) exponent. The geometrical fluctuations were found to be relevant if

$$
\phi>\frac{1-\alpha}{2-\alpha} .
$$

Although explicit calculations of $\phi$ have not been carried out for models with quenched connectivity disorder, simulations have shown that it appears to be remarkably difficult to escape from pure fixed points with such disorder, in contrast to the case of bond disorder. A prime example of this generic behaviour is Poisonnian (or Voronoi) random lattices, which have been shown to display the pure critical exponents for the 3D Ising model [7] to a very high degree of accuracy. Numerous other systems with quenched connectivity disorder show similar, pure critical behaviour [8]. However, the quenched connectivity disorder manifested by an ensemble of planar, trivalent random graphs, which we denote $\Phi^{3}$ graphs for brevity, does appear to give rise to new disordered fixed points [9]. The exponents of the Ising and $q \leqslant 4$ state Potts models, which already possess continuous transitions on flat 2D lattices, are altered and the first-order transition of higher-state Potts models is softened to a continuous transition $[9,10]$ on the $\Phi^{3}$ graphs.

The $\Phi^{3}$ graphs in question are precisely those generated in a simulation of pure 2D quantum gravity, though in that case one has an annealed ensemble in which the connectivities fluctuate. For such an annealed ensemble of $\Phi^{3}$ graphs the KPZ formula [11] shows how the conformal weights of operators in spin models living on the graphs are transformed from their flat $2 \mathrm{D}$ lattice values. The KPZ formula per se thus applies to systems in which the spins are fluctuating on the same timescale as the connectivity. One can, however, obtain predictions for the critical exponents of spin models living on an quenched ensemble of such graphs $\dagger$, which is what we are considering here, by taking a quenched limit in the KPZ formula [12-14]. In this limit one finds that the flat lattice conformal weight $\Delta$ is transmuted to a new quenched weight

$$
\tilde{\Delta}_{\text {quenched }}=\frac{\sqrt{1+24 \Delta}-1}{4}
$$

which may then be used to determine the critical exponents $\alpha$ and $\beta$ for the quenched ensemble $\$$.

$\dagger$ In which the connectivity disorder is frozen in.

$\ddagger$ It is only fair to remark that the analysis of the data in [9], on which the current paper is also based, only lends rather weak support to these predictions. 
To date, investigations of non-self-averaging behaviour in the presence of quenched disorder have concentrated exclusively on static quantities such as the susceptibility, rather than the dynamical properties described by the autocorrelation times for various observables. In this paper we study the non-self-averaging properties of autocorrelation times for the energy and the magnetization in the presence of quenched (in our case connectivity) disorder. We consider the Ising, $q=4$ and $q=10$ state Potts models on quenched ensembles of $\Phi^{3}$ random graphs, since the simulations of [9] provided strong evidence for new, disordered fixed points in all these cases.

In the next section we briefly recall the models studied and describe the Monte Carlo simulations performed. The results of the autocorrelation analyses are presented in section 3, and in section 4 we close with a summary and a few concluding remarks.

\section{The model and simulations}

As in [9] we use the standard definition of the $q$-state Potts model partition function and energy

$$
Z_{\text {Potts }}=\sum_{\left\{\sigma_{i}\right\}} \mathrm{e}^{-\beta E} \quad E=-\sum_{\langle i j\rangle} \delta_{\sigma_{i} \sigma_{j}} \quad \sigma_{i}=1, \ldots, q
$$

where $\beta=J / k_{\mathrm{B}} T$ is the inverse temperature in natural units, $\delta$ is the Kronecker symbol, and $\langle i j\rangle$ denotes the nearest-neighbour bonds of the random $\Phi^{3}$ graphs (without tadpoles or selfenergy bubbles) with $N$ sites. We consider the cases $q=2$ and 4 with $N=500,1000,2000$, $3000,4000,5000$, and 10000 which in the pure model exhibit second-order phase transitions, and the case $q=10$ with $N=250,500,1000,2000,3000,5000$, and 10000 which in the pure model undergoes a first-order phase transition.

The simulations were carried out using the Wolff single-cluster update algorithm [15]. For each lattice size we generated 64 independent graphs using the Tutte algorithm [16], and performed 500 thousand equilibration sweeps followed by up to 10 million measurement sweeps in order to obtain up to 500 thousand (almost) independent measurements for each lattice size. The runs were carried out at several $\beta$ values near the transition point and the time series of the energy $E$ and the magnetization $\dagger M=\left(q \max \left\{n_{i}\right\}-N\right) /(q-1)$ recorded for each graph. The inverse simulation temperatures $\beta_{\text {run }}$ are given in tables $1-3$. They should be compared with the recent estimates of $\beta_{c}=1.558(3), 1.835(1)$, and 2.244(1) for the transition points in the $q=2,4$, and 10 state quenched Potts models [9]. In what follows the per-site quantities are denoted by $e=E / N$ and $m=M / N$, the thermal averages on each individual graph by $\langle\ldots\rangle$ and the quenched average over the different graphs by $[\ldots]_{\mathrm{av}}$. From the time series of $e$ and $m$ it is straightforward to compute in the finite-size scaling (FSS) region various quantities at nearby values of $\beta$ by standard re-weighting [17] techniques.

To estimate the statistical (thermal) errors for each of the 64 realizations, the time-series data was split into bins, which were jack-knifed [18] to decrease the bias in the analysis of re-weighted data. The final values are averages over the 64 realizations which will be denoted by square brackets $[\ldots]_{\mathrm{av}}$, and the error bars are computed from the fluctuations among the realizations. Note that these errors contain both the average thermal error for a given realization and the theoretical variance for infinitely accurate thermal averages which is caused by the variation over the random graphs.

From the time series of the energy measurements we computed by re-weighting the average energy, the specific heat, and energetic fourth-order cumulant, as discussed in more detail in [9]. Similarly, we derived from the magnetization measurements the average magnetization, susceptibility, and magnetic cumulants and also evaluated mixed quantities involving both the

$\dagger$ Where $n_{i} \leqslant N$ denotes the number of spins of 'orientation' $i=1, \ldots, q$ in one lattice configuration. 
Table 1. $q=2$ autocorrelation times of the energy $\left(f=n_{\text {flip }}\langle|C|\rangle / N\right)$.

\begin{tabular}{rrrllllllll}
\hline \multicolumn{1}{r|}{$N$} & $\beta_{\text {run }}$ & $n_{\text {flip }}$ & {$[\langle|C|\rangle]$} & {$[\hat{\tau}]$} & {$[f][\hat{\tau}]$} & {$[\tau]=[f \hat{\tau}]$} & $\tau_{\min }$ & $\tau_{\max }$ & $\Delta \tau$ & $\Delta \tau /[\tau]$ \\
\hline 500 & 1.50 & 8 & $172.4(2.8)$ & $1.18(2)$ & 3.25 & $3.21(3)$ & 2.74 & 3.78 & 0.2069 & 0.0645 \\
1000 & 1.50 & 12 & $258.8(4.6)$ & $1.15(2)$ & 3.56 & $3.50(3)$ & 2.79 & 4.04 & 0.2193 & 0.0626 \\
2000 & 1.50 & 20 & $386.0(8.0)$ & $1.00(2)$ & 3.87 & $3.79(4)$ & 3.32 & 4.45 & 0.2463 & 0.0650 \\
3000 & 1.55 & 12 & $896(20)$ & $1.09(3)$ & 3.90 & $3.80(4)$ & 3.39 & 4.29 & 0.2584 & 0.0679 \\
4000 & 1.55 & 10 & $1076(21)$ & $1.39(3)$ & 3.74 & $3.66(4)$ & 2.96 & 4.22 & 0.1178 & 0.0644 \\
5000 & 1.55 & 8 & $1305(26)$ & $1.83(4)$ & 3.83 & $3.74(3)$ & 3.08 & 4.49 & 0.2426 & 0.0649 \\
10000 & 1.55 & 10 & $2328(53)$ & $1.75(5)$ & 4.07 & $3.94(3)$ & 3.38 & 4.48 & 0.2316 & 0.0587 \\
\hline
\end{tabular}

Table 2. $q=4$ autocorrelation times of the energy $\left(f=n_{\text {flip }}\langle|C|\rangle / N\right)$.

\begin{tabular}{rrrllllllll}
\hline$N$ & $\beta_{\text {run }}$ & $n_{\text {flip }}$ & {$[\langle|C|\rangle]$} & {$[\hat{\tau}]$} & {$[f][\hat{\tau}]$} & {$[\tau]=[f \hat{\tau}]$} & $\tau_{\min }$ & $\tau_{\max }$ & $\Delta \tau$ & $\Delta \tau /[\tau]$ \\
\hline 500 & 1.84 & 8 & $221.9(4.7)$ & $3.54(07)$ & 12.58 & $12.32(14)$ & 9.77 & 14.67 & 1.1122 & 0.0903 \\
1000 & 1.84 & 12 & $371.1(8.9)$ & $3.22(07)$ & 14.32 & $13.89(13)$ & 11.72 & 16.11 & 1.0344 & 0.0745 \\
2000 & 1.84 & 16 & $639(19)$ & $3.24(10)$ & 16.55 & $15.74(16)$ & 12.48 & 18.93 & 1.2805 & 0.0814 \\
3000 & 1.84 & 16 & $909(27)$ & $3.55(11)$ & 17.18 & $16.37(21)$ & 13.30 & 21.06 & 1.6273 & 0.0994 \\
4000 & 1.84 & 18 & $1093(28)$ & $3.56(11)$ & 17.54 & $16.83(20)$ & 12.55 & 20.66 & 1.5668 & 0.0931 \\
5000 & 1.85 & 20 & $1634(40)$ & $2.71(08)$ & 17.73 & $16.99(22)$ & 14.12 & 22.90 & 1.6845 & 0.0991 \\
10000 & 1.85 & 20 & $3194(81)$ & $2.87(11)$ & 18.36 & $17.37(23)$ & 13.58 & 22.76 & 1.8138 & 0.1044 \\
\hline
\end{tabular}

Table 3. $q=10$ autocorrelation times of the energy $\left(f=n_{\text {flip }}\langle|C|\rangle / N\right)$.

\begin{tabular}{rlccccccccc}
\hline$N$ & $\beta_{\text {run }}$ & $n_{\text {flip }}$ & {$[\langle|C|\rangle]$} & {$[\hat{\tau}]$} & {$[f][\hat{\tau}]$} & {$[\tau]=[f \hat{\tau}]$} & $\tau_{\min }$ & $\tau_{\max }$ & $\Delta \tau$ & $\Delta \tau /[\tau]$ \\
\hline 250 & 2.20 & 6 & $67.8(2.1)$ & $40.3(1.9)$ & 65.7 & $66.0(4.0)$ & 23.6 & 176.2 & 31.63 & 0.4796 \\
500 & 2.20 & 6 & $83.8(3.8)$ & $74.4(3.2)$ & 74.9 & $75.5(4.9)$ & 22.1 & 244.1 & 39.10 & 0.5179 \\
1000 & 2.20 & 6 & $86.2(3.6)$ & $134.9(5.4)$ & 69.8 & $70.2(4.2)$ & 23.6 & 175.2 & 33.34 & 0.4750 \\
2000 & 2.22 & 12 & $189(14)$ & $111.9(5.2)$ & 127 & $127(12)$ & 34.9 & 555.5 & 90.89 & 0.7163 \\
3000 & 2.23 & 15 & $362(29)$ & $109.5(4.7)$ & 198 & $185(13)$ & 54.6 & 528.1 & 97.01 & 0.5252 \\
5000 & 2.24 & 15 & $833(52)$ & $136.0(6.9)$ & 340 & $308(18)$ & 85.3 & 761.1 & 144.7 & 0.4699 \\
10000 & 2.242 & 15 & $1652(110)$ & $210(11)$ & 521 & $452(27)$ & 156.0 & 1159.2 & 213.6 & 0.4730 \\
\hline
\end{tabular}

energy and magnetization. However, it is the dynamical aspects of the simulations which are our principal concern here and these are characterized by the autocorrelation functions and the associated integrated autocorrelation times $\hat{\tau} \dagger$. It is now customary when discussing single-cluster algorithm simulations [19] to convert the $\hat{\tau}$ thus obtained by multiplying with a factor $f=n_{\text {flip }}\langle|C|\rangle / N$ to a standardized scale where, on average, measurements are taken after every spin has been flipped once. Here $\langle|C|\rangle$ is the average cluster size and $n_{\text {flip }}$ denotes the number of cluster flips between measurements. The thus obtained $\tau=f \hat{\tau}$ allows a fair comparison with, e.g., Metropolis simulations.

When one has quenched random disorder this procedure is not unique due to the average over realizations $\left([\ldots]_{\mathrm{av}}\right)$, since one can take either $[\tau]_{\mathrm{av}} \equiv[f \cdot \hat{\tau}]_{\mathrm{av}}$ or $[f]_{\mathrm{av}} \cdot[\hat{\tau}]_{\mathrm{av}}$. We have presented the raw data and both variations in tables 1-3 for the energy for $q=2,4$, and 10 where it can be seen that the differences between the two averaging prescriptions are rather small, so for all practical purposes they can be considered to be equivalent. The magnetization data is qualitatively similar and is not reproduced here. For definiteness in the scaling analysis

$\uparrow$ The exponential autocorrelation time $\hat{\tau}_{\exp }$ for an observable $X$ is defined by the exponential decay of different time correlators $A(t) \equiv(\langle X(t) X(0)\rangle-\langle X(t)\rangle\langle X(0)\rangle) /\left(\left\langle X(0)^{2}\right\rangle-\langle X(0)\rangle^{2}\right) \sim \exp \left(-t / \hat{\tau}_{\text {exp }}\right)$ for large time differences $t$ and the associated integrated autocorrelation time $\hat{\tau}$ by (formally) $\int_{0}^{\infty} \mathrm{d} t A(t) \sim \hat{\tau}$. 
we take $[\tau]_{\mathrm{av}} \equiv[f \cdot \hat{\tau}]_{\mathrm{av}}$. In tables $1-3$ the minimum value of $\tau(=f \cdot \hat{\tau})$ for the various realizations is denoted by $\tau_{\min }$ and the maximum by $\tau_{\max }$. The standard deviation $\Delta \tau$ and its scaled form, $\Delta \tau /[\tau]_{\mathrm{av}}$, are also tabulated.

\section{Results for autocorrelation times}

The integrated autocorrelation times for each random-graph realization are obtained by blocking techniques $\dagger$. These are (necessarily) measurements at the simulation points which are chosen close to a FSS sequence of $\beta$-values but not with high precision since these are a priori estimates based on the results on smaller lattices. For higher accuracy one would have to redo the simulations using the knowledge of the infinite-volume estimates of $\beta_{c}$ or the locations of the maxima of $C, \chi$, etc, obtained from the present batch of runs and presented in [9]. As a consequence of this choice of measurement points it can be seen in what follows that one or two data points lie rather far from the general trend.

Looking at the behaviour of the autocorrelation times for each $q$ in turn, we can see that the autocorrelation times for $q=2$ stay roughly constant with increasing system size for both the energy and the magnetization. For the energy we obtain values in the range 3-4, and for the magnetization in the range 1.6-2.2. These results are obtained with the cluster update for general $q$-state Potts models where the new spin direction $s_{\text {new }}$ for a cluster is chosen randomly from $s_{\text {new }} \in[1, q]$. Picking the old value, $s_{\text {new }}=s_{\text {old }}$, would not change anything. Hence for $q=2$ the autocorrelation time can be reduced by a factor of 2 by requiring that the spin direction of the cluster flips rather than is chosen randomlył.

For $q=4$ the autocorrelation times are also still reasonably small, covering a range 12-18 for the energy and a range 7-10 for the magnetization. However, here a scaling with system size is now clearly observable and fits to the standard FSS ansatz $§$

$$
[\tau]_{\mathrm{av}}=a N^{z / D}
$$

give for the energy $\ln a=2.283(82)$ and $z / D=0.064(10)$ with $\chi^{2} /$ dof $=1.28$ or a goodness-of-fit parameter $Q=0.28$, if the $N=500$ and $N=1000$ graphs are omitted. For the magnetization the fit through all available graph sizes yields $\ln a=1.554$ (95) and $z / D=0.074(13)$ with $\chi^{2} /$ dof $=1.01$ or $Q=0.41$. The data along with the fits are shown in figure $1(a)$. Note that the estimates of $z / D$ on the random graphs are considerably smaller than for regular lattices [20] where $z / D=0.876(11) / 2=0.438(6)$ for the integrated autocorrelation time of the energy, using the Swendsen-Wang cluster-update algorithm $\|$. In fact, since the Li-Sokal bound [21] guarantees for regular lattices that $\tau_{e} \geqslant$ const $\times C$ and the specific heat $C$ diverges on regular lattices at criticality like $L(\ln L)^{-3 / 2}$, we see that (as was noted in [20]) this actually must be an underestimate. It should be emphasized that for quenched (or annealed) gravity graphs the singularity of the specific heat is predicted to be weakened; hence we are not a priori in contradiction with the suitably generalized Li-Sokal bound.

For $q=10$, where one would expect on regular lattices for both autocorrelation times a pronounced exponential increase with system size due to the first-order nature of the transition, the first point to note is that here on $\Phi^{3}$ graphs the values are very large in comparison with the

$\dagger$ In practice a convenient method of obtaining integrated autocorrelation times is to note that the naive variance of the mean for time-series data calculated from each individual measurement will be a factor of $2 \hat{\tau}$ smaller than the variance calculated by blocking the data into bins of length $\gg \hat{\tau}$.

$\ddagger$ For $N=4000$ the data in table 1 are rescaled to the 'Potts units'.

$\S$ Here $D(\approx 4)$ is the fractal dimension of the graphs, but in the following we shall not need this number explicitly.

$\|$ In two dimensions the difference between the dynamical critical exponent of the Wolff single-cluster and SwendsenWang cluster algorithm, if any, is empirically extremely small. 

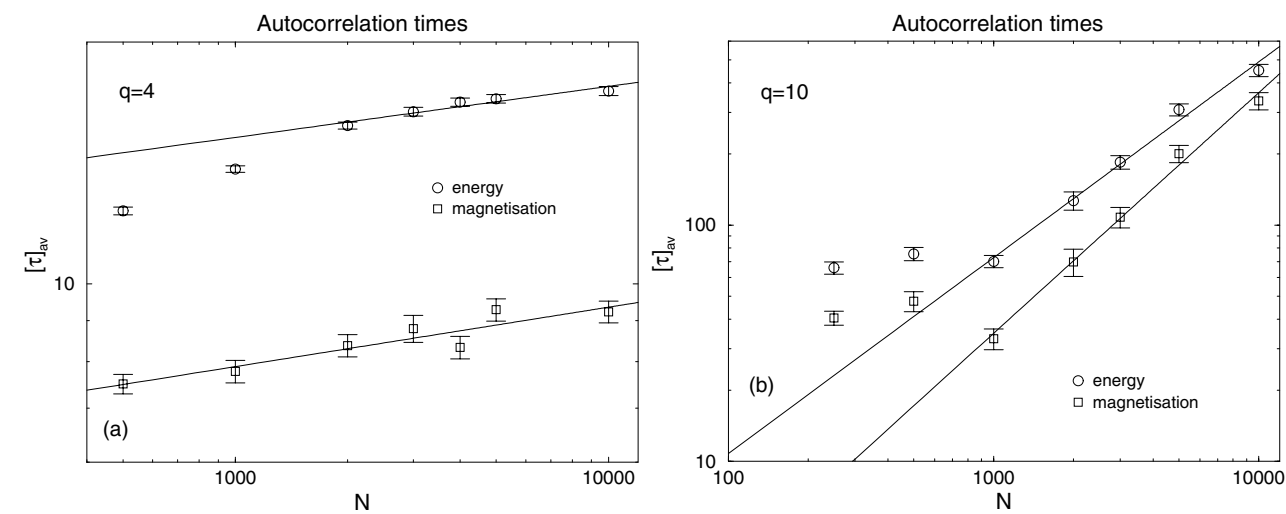

Figure 1. The data points and fits to $[\tau]_{\mathrm{av}}=a N^{z / D}$ for both the energy and magnetization in (a) the $q=4$ and (b) the $q=10$ Potts model.
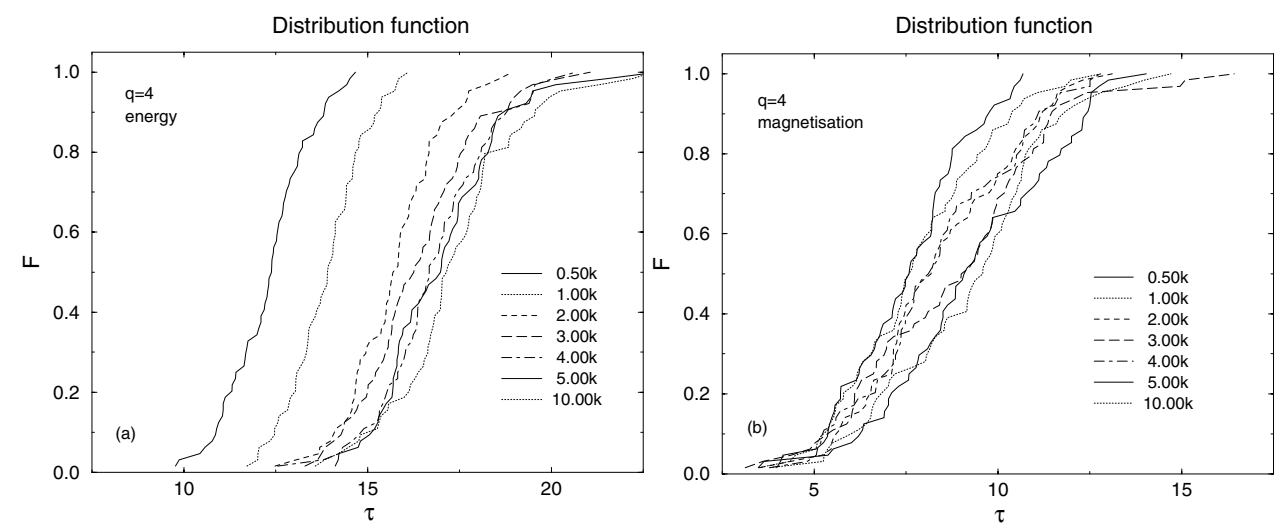

Figure 2. The cumulative distribution function of autocorrelation times for $(a)$ the energy and (b) the magnetization in the $q=4$ Potts model. The system size is increasing as the curves move to the right.

Ising and $q=4$ Potts model measurements discussed above, falling into the range 60-500 for the energy, and 40-350 for the magnetization. The increase of $[\tau]_{\mathrm{av}}$ with system size, however, is consistent with a power-law scaling behaviour rather than the exponential increase of a firstorder transition; see figure $1(b)$. If we omit the two smallest graph sizes with $N=250$ and $N=500$ we obtain from fits of the energy autocorrelations the estimates $\ln a=-1.44(29)$ and $z / D=0.829(35)$ with $\chi^{2} /$ dof $=1.97$ or $Q=0.12$. Fits of the magnetization autocorrelations yield $\ln a=-3.49(44)$ and $z / D=1.019(54)$ with $\chi^{2} / \operatorname{dof}=1.00$ or $Q=0.39$.

For quenched, random systems not only is the scaling behaviour of the average $[\tau]_{\mathrm{av}}$ of interest but also the properties of the whole probability density $P(\tau)$. Since we have only few (=64) events for sampling this density it is numerically (and mathematically) more sensible to consider the cumulative probability distribution $F(\tau)=\int_{0}^{\tau} P\left(\tau^{\prime}\right) \mathrm{d} \tau^{\prime}$, with the obvious relation $\mathrm{d} F(\tau) / \mathrm{d} \tau=P(\tau)$.

In figure 2 we have plotted the cumulative distribution of the autocorrelation times $\tau=f \cdot \hat{\tau}$ at the simulation point in the $q=4$ model for both the energy and the magnetization. It is clear from the graph that the distribution is broadening with increasing lattice size, rather than 


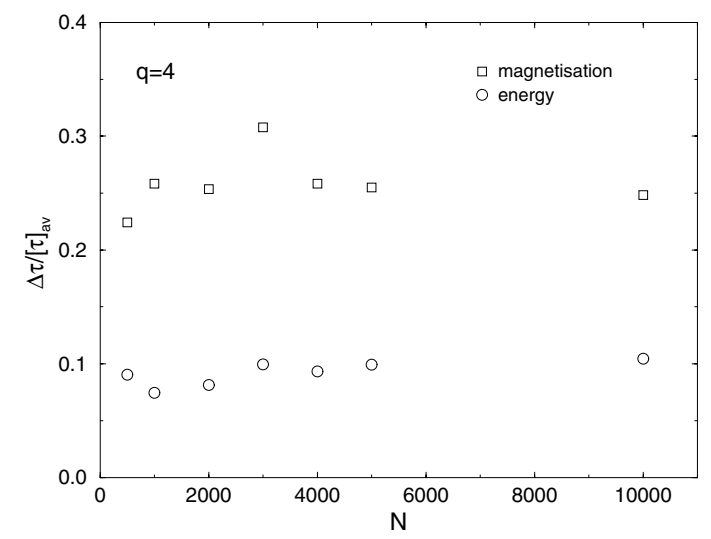

Figure 3. A plot of $\Delta \tau /[\tau]_{\text {av }}$ for both the energy and magnetization in the $q=4$ Potts model. Given the caveat in the text regarding the simulation points this is clearly tending to a constant for increasing system size.

sharpening. For self-averaging measurements one would expect the curves to tend to a step function, since the underlying probability density of $\tau$ would tend to a delta-function in such a case. One can therefore conclude that the measurements of $\tau$ in the simulations provide strong evidence for non-self-averaging behaviour in the $q=4$ model. This statement can be made more quantitative by considering similarly to equation (1) the ratio of the width of the probability density, the standard deviation $\Delta \tau$, and the average value, $[\tau]_{\mathrm{av}}$, which sets the scale. As this is a property of the quenched randomness here we tacitly assume that the thermal noise of the estimates of $\tau$ for a given realization can be neglected which, in view of our extremely high statistics, is justified.

The plot of $\Delta \tau /[\tau]_{\mathrm{av}}$ in figure 3 shows that the relative widths of the densities of both the energy and the magnetization stay roughly constant with increasing system size, thus clearly demonstrating the lack of self-averaging. Another way to demonstrate this property graphically is to plot the probability distribution $F$ against the scaled variable $\tau /[\tau]_{\text {av }}$. If the density is non-self-averaging with $\Delta \tau /[\tau]_{\mathrm{av}}=$ const one expects to see in such a plot data collapse onto a single master-curve. As can be seen in figure 4 this is indeed the case for both the energy and the magnetization.

For $q=2$ the corresponding plots look very similar and are not reproduced here. More interesting is the qualitatively different case of the $q=10$ Potts model, because here the first-order transition on regular lattices is softened to a second-order transition for quenched, random graphs. Here the plots of the cumulative distributions shown in the insets of figure 5 again clearly exhibit the broadening with increasing lattice size just as for $q=4$, rather than sharpening. Therefore it is even more impressive to observe that replotting the data versus the scaled variable $\tau /[\tau]_{\text {av }}$ still produces well-defined master curves for both the energy and the magnetization as can be seen in figure 5. The scaling of $\Delta \tau /[\tau]_{\text {av }}$ as a function of system size looks slightly more scattered than for the $q=4$ data in figure 3 (recall that the effective statistics per graph is smaller by about one order of magnitude compared with the $q=4$ model), but here also it is safe to claim that $\Delta \tau /[\tau]_{\mathrm{av}}$ stays roughly constant with increasing system size. One can therefore conclude that the measurements of $\tau$ in the simulations provide strong evidence for non-self-averaging behaviour in the $q=10$ model as well. 

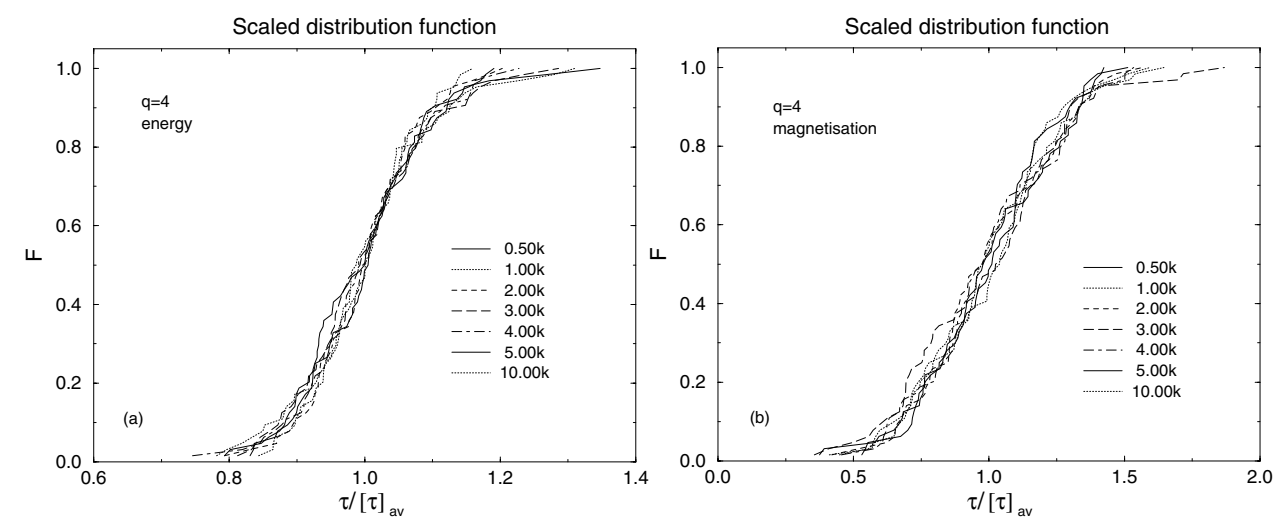

Figure 4. The scaled cumulative distribution function of autocorrelation times for $(a)$ the energy and $(b)$ the magnetization in the $q=4$ Potts model showing the good data collapse.
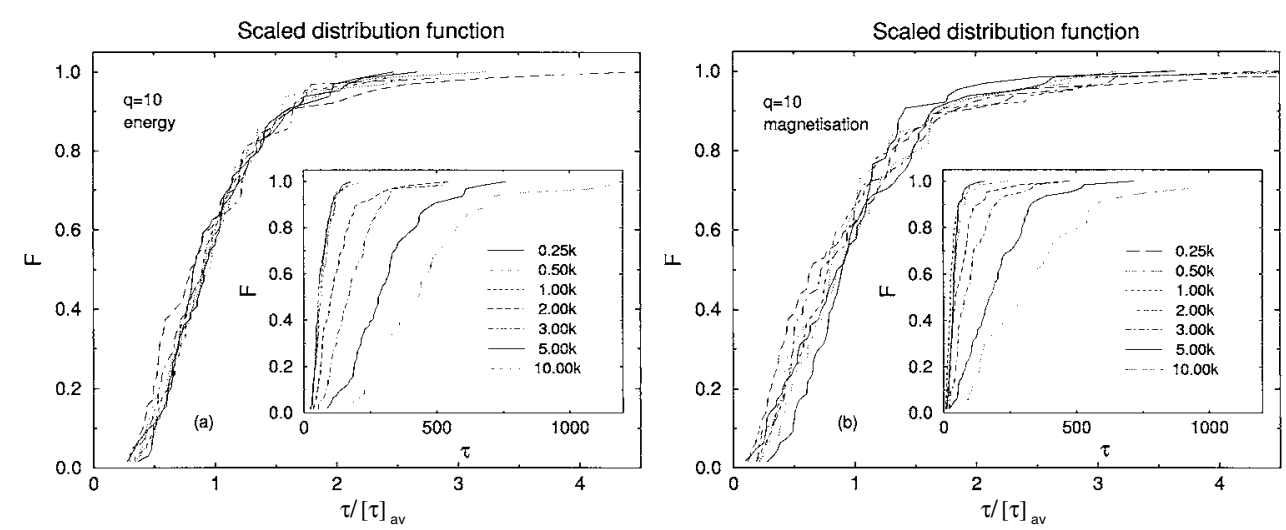

Figure 5. The scaled cumulative distribution function of autocorrelation times for $(a)$ the energy and $(b)$ the magnetization in the $q=10$ Potts model. The insets show the original data. The system size is increasing as the curves move to the right.

\section{Conclusions}

Our previous analysis of the static properties of simulations of the Ising and $q=4,10$ state Potts models on $\Phi^{3}$ graphs showed that the quenched connectivity disorder they possessed altered the exponents of models with a continuous transition on a regular lattice, and softened the first-order transition of the $q=10$ model to a continuous transition.

The analysis of the autocorrelation times discussed here shows that these models display another property that is often associated with a disordered fixed point with a distribution of couplings, namely, non-self-averaging. One must, however, be a little careful in using non-selfaveraging as a diagnostic for distinguishing pure and disordered fixed points: the Ising model on a 3D Poisonnian random lattice has been shown to have the standard critical exponents to a very high degree of accuracy, but nonetheless still displays non-self-averaging of both static and dynamical properties [7]. On the basis of these observations in [7] non-self-averaging is not restricted to disordered fixed points alone.

Whatever the circumstances in which non-self-averaging appears the analysis here shows that it is also manifest in the autocorrelation times of the systems in question and amenable to 
a quantitative scaling analysis. Although we have only discussed the Ising and $q=4,10$ state Potts models on $\Phi^{3}$ random graphs here, it is clear that the idea of looking at the probability density $P(\tau)$ or rather the cumulative probability distribution function $F(\tau)$ and studying its scaling properties to discern non-self-averaging is generally applicable.

\section{Acknowledgments}

DJ was partially supported by a Royal Society of Edinburgh/SOEID Support Research Fellowship. WJ acknowledges partial support by the German-Israel-Foundation (GIF) under contract No I-0438-145.07/95. The collaborative work of DJ and WJ was funded by ARC grant 313-ARC-XII-98/41, the EC IHP network 'Discrete Random Geometries: From Solid State Physics to Quantum Gravity' HPRN-CT-1999-000161 and the ESF network 'Geometry and Disorder: From Membranes to Quantum Gravity'. The numerical simulations were performed on a T3D parallel computer of Zuse-Zentrum für Informationswissenschaften Berlin (ZIB) under grant No bvpf01.

\section{References}

[1] For a recent review see Cardy J 1999 Quenched randomness at first-order transitions STATPHYS20 (Paris, July 1998) Physica A 263215

(Cardy J 1998 Preprint cond-mat/9806355)

[2] Harris A B 1974 J. Phys. C: Solid State Phys. 71671

Imry Y and Wortis M 1979 Phys. Rev. B 193581

Aizenman M and Wehr J 1989 Phys. Rev. Lett. 622503

Hui K and Berker A N 1989 Phys. Rev. Lett. 622507

Hui K and Berker A N 1989 Phys. Rev. Lett. 632433 (erratum)

[3] Aharony A and Harris A B 1996 Phys. Rev. Lett. 773700

[4] Wiseman S and Domany E 1998 Phys. Rev. E 582938

[5] Wiseman S and Domany E 1995 Phys. Rev. E 523469

[6] Luck J M 1993 Europhys. Lett. 24359

[7] Janke W and Villanova R 2000 in preparation

[8] Janke W, Katoot M and Villanova R 1993 Phys. Lett. B 315412

Janke W, Katoot M and Villanova R 1994 Nucl. Phys. B (Proc. Suppl.) 34698

Janke W, Katoot M and Villanova R 1994 Phys. Rev. B 499644

Janke W and Villanova R 1995 Phys. Lett. A 209179

Janke W, Katoot M and Villanova R 1996 Nucl. Phys. B (Proc. Suppl.) 47641

[9] Janke W and Johnston D 2000 Nucl. Phys. B at press

(Janke W and Johnston D 1999 Preprint hep-lat/9907026, Leipzig LU-ITP 1999/013 and NTZ 17/1999)

[10] Baillie C, Janke W and Johnston D 1996 Phys. Lett. B 38814

Baillie C, Janke W and Johnston D 1997 Nucl. Phys. B (Proc. Suppl.) 53732

Baillie C F, Hawick K A and Johnston D A 1994 Phys. Lett. B 328284

[11] Knizhnik V G, Polyakov A M and Zamolodchikov A B 1998 Mod. Phys. Lett. A 3819

David F 1988 Mod. Phys. Lett. A 31651

Distler J and Kawai H 1989 Nucl. Phys. B 321509

[12] Cardy J 1999 J. Phys. A: Math. Gen. 32 L177

[13] Johnston D A 1992 Phys. Lett. B 277405

[14] Janke W and Johnston D A 1999 Phys. Lett. B 460271

[15] Wolff U 1989 Phys. Rev. Lett. 62361

Wolff U 1990 Nucl. Phys. B 334581

[16] Agishtein M E and Migdal A A 1991 Nucl. Phys. B 350690

Tutte W 1962 Can. J. Math. 1421

Tutte W 1963 Can. J. Math. 15249

[17] Ferrenberg A M and Swendsen R H 1988 Phys. Rev. Lett. 612635

Ferrenberg A M and Swendsen R H 1989631658 (erratum)

[18] Miller R G 1974 Biometrika 611 
Efron B 1982 The Jackknife, the Bootstrap and other Resampling Plans (Philadelphia: SIAM)

[19] Wolff U 1989 Phys. Rev. Lett. 62361

Wolff U 1990 Nucl. Phys. B 334581

[20] Salas J and Sokal A D 1997 J. Stat. Phys. 88567

[21] Li X-J and Sokal A D 1989 Phys. Rev. Lett. 63827 